\title{
Monte Carlo simulation results for critical Casimir forces
}

\author{
O. Vasilyev, ${ }^{1,2}$ A. Gambassi, ${ }^{1,2}$ A. Maciołek, ${ }^{1,2,3}$ and S. Dietrich ${ }^{1,2}$ \\ ${ }^{1}$ Max-Planck-Institut für Metallforschung, \\ Heisenbergstr. 3, 70569 Stuttgart, Germany \\ ${ }^{2}$ Institut für Theoretische und Angewandte Physik, \\ Universität Stuttgart, Pfaffenwaldring 57, D-70569 Stuttgart, Germany. \\ ${ }^{3}$ Institute of Physical Chemistry, Polish Academy of Sciences, \\ Kasprzaka 44/52, 01-224 Warsaw, Poland.
}

\begin{abstract}
The confinement of critical fluctuations in soft media induces critical Casimir forces acting on the confining surfaces. The temperature and geometry dependences of such forces are characterized by universal scaling functions. A novel approach is presented to determine them for films via Monte Carlo simulations of lattice models. The method is based on an integration scheme of free energy differences. Our results for the Ising and the XY universality class compare favourably with corresponding experimental results for wetting layers of classical binary liquid mixtures and of ${ }^{4} \mathrm{He}$, respectively.
\end{abstract}




\section{INTRODUCTION}

Recent progress in understanding the features of effective forces induced by confined fluctuations, both quantum and thermal, reveals the potential relevance of these so-called Casimir forces [1] for numerous applications, ranging from microelectromechanical systems (MEMS) to the physics of colloids [2, 3, 4] . Thermal fluctuation-induced Casimir forces $f_{\mathrm{C}}$ acting on the confining surfaces of fluids near critical points [5] are of particular interest because they become largely independent of the microscopic details of the system, acquiring a universal character [5, 6, 7], and they can be switched on and off upon varying, e.g., the temperature. Moreover, by changing the surface chemistry they can be relatively easily turned from attractive to repulsive [4], in contrast to the Casimir force stemming from electromagnetic fluctuations, for which such a possibility is currently debated as being very desirable to avoid stiction in MEMS, but difficult to achieve. Finite-size scaling theory (see, e.g., ref. [3]) predicts that the temperature dependence of the critical Casimir force $f_{\mathrm{C}}$ is described by universal scaling functions which depend on the bulk universality class (UC) of the confined medium and on the surface UCs of the confining surfaces [6]. The latter are related to the boundary conditions (BC) [3, 6] imposed by the surfaces on the relevant fluctuating field, i.e., the order parameter (OP) of the underlying second-order phase transition. In spite of intensive theoretical and experimental efforts, the current knowledge of these scaling functions is still rather limited even for relevant UCs such as the Ising one, which characterizes the critical behaviour of simple fluids and binary liquid mixtures. In three spatial dimensions (3D) the only available results refer, theoretically, to films with periodic BC (PBC), investigated via Monte Carlo (MC) simulations [8], or field-theoretical methods (Dirichlet, Neumann BC, PBC) [7], and, experimentally, to complete wetting films of binary liquid mixture [9] belonging to the surface UC characterized by symmetry-breaking surface fields [6]. The corresponding BC (+-) of opposing surface fields reflect the fact that the two confining surfaces exhibit opposite adsorption preferences for the two species of the mixture. At the bulk critical point the dependence of $f_{\mathrm{C}}$ on the thickness $L$ of the film turned out to be in good agreement with the corresponding theoretical predictions [10, 11]. However, the determination of the full temperature dependence of $f_{\mathrm{C}}$ from these very difficult experiments suffers from significant statistical and systematic uncertainties, enhancing the need of theoretical insight. Indeed, several features of the associated scaling function, such 
as its global shape and its dependence on the spatial dimensionality $d$ and BC still await theoretical investigations. Exact results are available in $d=2$ [12] and $d \geq 4$ [10] (meanfield theory) both for $(+-)$ and $(++) \mathrm{BC}$, the latter corresponding to the case in which both confining surfaces exhibit preference for the same species of the mixture. Proposals to measure the temperature dependence of the Casimir force between a colloid and a flat surface or between colloidal particles dissolved in a near-critical binary liquid mixture [4] call for a detailed theoretical analysis of the associated scaling behaviour. The relevant missing pieces, mentioned above, in the theoretical analysis of the scaling behaviour of $f_{\mathrm{C}}$ require to account for the fluctuations, including the dimensional crossover occurring in a film. This is a rather challenging task, especially if the OP profile is inhomogeneous across the film, as in the cases we are interested in. With these elements out of reach of current analytical techniques, MC simulations provide a useful alternative approach. The available $\mathrm{MC}$ results for the $d=3$ Ising model are restricted to the case of PBC [8], in which the scaling function of $f_{\mathrm{C}}$ can be determined - up to a normalization factor - by numerically measuring the expectation value of a suitable lattice stress tensor. The purpose of the present contribution is to present a novel approach for the MC simulation of the Casimir force and to provide data for the scaling behaviour of $f_{\mathrm{C}}$. We focus on the Ising $\mathrm{UC}$ with the experimentally relevant $\mathrm{BC}(++)$ and $(+-)$. We also compare our results with those in ref. [8], providing an independent test of the method proposed therein. Our method is based on an integration scheme of free energy differences and it has the advantage, compared to the latter, of providing the absolute value for $f_{\mathrm{C}}$ and of being applicable for arbitrary BC. The comparison with the experimental data in ref. [9] reveals good agreement.

Measurements [13] of the equilibrium thickness of ${ }^{4} \mathrm{He}$ wetting films near the superfluid temperature $T_{\lambda}$ provide an experimental determination of the scaling function of $f_{\mathrm{C}}$ for the XY UC with Dirichlet BC on both surfaces, corresponding to the so-called ordinary surface UC [6]. These BC are due to the fact that the superfluid OP vanishes at the surfaces. In this case more analytical and numerical results are available. For temperatures $T \geq T_{\lambda}$ fieldtheoretical calculations [7] of the scaling function are in agreement with the experimental data [13], whereas its behaviour for $T \ll T_{\lambda}$ has been determined by accounting for Hespecific features related to capillary-wavelike surface fluctuations [14]. In addition, valuable information on the shape of the scaling function in the critical region has been obtained on the basis of Landau-Ginzburg theory [15]. Recent MC simulations [16] have nicely confirmed 
and extended the available analytic and experimental results. Since our approach differs from the one in ref. [16] we also present our results for the scaling function in this case, providing a valuable test.

In a film geometry with thickness $L$ and large transverse area $A$, the Casimir force $f_{\mathrm{C}}$ per unit area $A$ and in units of $k_{\mathrm{B}} T \equiv \beta^{-1}$ is defined as $f_{\mathrm{C}}(\beta, L) \equiv-\partial f^{\mathrm{ex}} / \partial L$, where $f^{\operatorname{ex}}(\beta, L) \equiv \beta L\left[f-f^{\text {bulk }}(\beta)\right]$ is the excess free energy (which depends on the BC), $f$ is the free energy of the film per unit volume $V=L A$ and $f^{\text {bulk }}$ is the bulk free energy density. According to finite-size scaling [3] the Casimir force takes the universal scaling form

$$
f_{\mathrm{C}}(\beta, L)=L^{-d} \vartheta\left(\tau\left(L / \xi_{0}^{+}\right)^{1 / \nu}\right)
$$

where the scaling function $\vartheta(x)$ depends on the $\mathrm{BC}, \tau=\left(\beta_{c}-\beta\right) / \beta$ is the reduced temperature and $\xi=\xi_{0}^{ \pm}|\tau|^{-\nu}$ is the bulk correlation length which controls the spatial exponential decay of correlations. The critical exponent $\nu$ equals 0.6301(4) and 0.662(7) for the 3D Ising and XY UCs, respectively [17]; $\xi_{0}^{ \pm}$are nonuniversal amplitudes above $(+)$and below $(-)$ $T_{c}$.

\section{COMPUTATION OF THE SCALING FUNCTIONS}

We compute the Casimir force for the Ising and XY models defined on a 3D simple cubic lattice in slab geometry $\left(L_{x} \times L_{y} \times L_{z}\right.$ with $L_{x}=L_{y} \gg L_{z} \equiv L$ and $\left.A=L_{x} \times L_{y}\right)$ via the Hamiltonian $H=-J \sum_{\langle i, j\rangle} \mathbf{s}_{i} \cdot \mathbf{s}_{j}$, where the sum $\langle i, j\rangle$ is taken over all nearest neighbour pairs of sites $i$ and $j$ on the lattice. In the Ising model, $\mathbf{s}_{i}$ has only one component $s_{i} \in\{+1,-1\}$, whereas in the XY model $\mathbf{s}_{i}$ is a two-component vector with modulus $\left|\mathbf{s}_{i}\right|=1$. With the Hamiltonian $H$ one finds $\beta_{c}=0.2216544(3)$ and $\xi_{0}^{+}=0.501(2)$ [18] for the Ising model, whereas $\beta_{c}=0.45420(2)$ and $\xi_{0}^{+}=0.498(2)$ [19], for the XY model. [23] Temperatures and energies are measured in units of $J$ and $\xi_{0}^{+}$in units of the lattice spacing. In the $x$ and $y$ directions we assume PBC whereas in the $z$ direction we consider periodic, free, and fixed BC (i.e., for the Ising model, $s_{i}=+1(+)$ or $s_{i}=-1(-)$ at the boundaries). For large $A$, the total free energy $F(\beta, L, A)$ of such systems decomposes as $F(\beta, L, A) \equiv A L f=$ $A\left[L f^{\text {bulk }}(\beta)+\beta^{-1} f^{\operatorname{ex}}(\beta, L)\right]$, where $f^{\operatorname{ex}}(\beta, L=\infty)$ is the contribution to $F$ due to the two

isolated surfaces, macroscopically far apart from each other, whereas $f^{\operatorname{ex}}(\beta, L)-f^{\operatorname{ex}}(\beta, \infty)$ is the $L$-dependent finite-size contribution we are interested in. On the lattice $\left(^{\wedge}\right), f_{\mathrm{C}}(\beta, L)$ 
is given by

$$
\hat{f}_{\mathrm{C}}\left(\beta, L-\frac{1}{2}, A\right) \equiv-\frac{\beta \Delta F(\beta, L, A)}{A}+\beta f^{\text {bulk }}(\beta),
$$

where $\Delta F(\beta, L, A)=F(\beta, L, A)-F(\beta, L-1, A)$.

In general MC methods do note lend themselves to the efficient computation of quantities such as $F$, which cannot be expressed as suitable ensemble averages. However, the free energy difference $\Delta F(\beta, L, A)$ we are interested in can be cast in such a form via the socalled "coupling parameter approach" (see, e.g., ref. [20]). This is a viable alternative to the method used in ref. [8] in which $\Delta F$ has been expressed as the ensemble average of a lattice stress tensor, which so far is only applicable for PBC. We consider two lattice models with the same configuration space $\mathcal{C}$ but different Hamiltonians $H_{1}$ and $H_{2}$, so that their free energies are given by $F_{i}=-\frac{1}{\beta} \ln \sum_{\mathcal{C}} \exp \left(-\beta H_{i}\right)$ where $\sum_{\mathcal{C}}$ indicates the sum over all possible configurations belonging to $\mathcal{C} . F_{2}-F_{1}$ can be conveniently computed by introducing the crossover Hamiltonian

$$
H_{\mathrm{cr}}(\lambda)=(1-\lambda) H_{1}+\lambda H_{2}
$$

which depends on the coupling parameter $\lambda \in[0,1]$ and interpolates between $H_{1}$ and $H_{2}$ as $\lambda$ increases from 0 to 1 . Accordingly, the free energy $F_{\mathrm{cr}}(\lambda)=-\frac{1}{\beta} \ln \sum_{\mathcal{C}} \exp \left(-\beta H_{\mathrm{cr}}(\lambda)\right)$ of a system with Hamiltonian $H_{\mathrm{cr}}(\lambda)$ and configuration space $\mathcal{C}$ interpolates between $F_{1}$ and $F_{2}$. The derivative of $F_{\mathrm{cr}}(\lambda)$ with respect to the coupling parameter,

$$
\frac{\mathrm{d} F_{\mathrm{cr}}(\lambda)}{\mathrm{d} \lambda}=\frac{\sum_{\mathcal{C}}\left(H_{2}-H_{1}\right) \mathrm{e}^{-\beta H_{\mathrm{cr}}(\lambda)}}{\sum_{\mathcal{C}} \mathrm{e}^{-\beta H_{\mathrm{cr}}(\lambda)}}=\langle\Delta H\rangle_{H_{\mathrm{cr}}(\lambda)},
$$

takes the form of the canonical ensemble average $\langle\ldots\rangle_{H_{\mathrm{cr}}(\lambda)}$ (with Hamiltonian $H_{\mathrm{cr}}(\lambda)$ ) of $\Delta H \equiv H_{2}-H_{1}$ and therefore it can be efficiently computed via MC simulations. A straightforward integration over $\lambda$ yields the expression for the free energy difference

$$
F_{2}-F_{1}=\int_{0}^{1} \mathrm{~d} \lambda\langle\Delta H\rangle_{H_{\mathrm{cr}}(\lambda)} \equiv I
$$

in terms of an ensemble average (see, e.g., ref. [20]).

The Casimir force is related to the difference $\Delta F(\beta, L, A)$ (see eq. (2)) between the free energies $F(\beta, L, A)$ and $F(\beta, L-1, A)$ of the same model on two lattices with different numbers of sites and therefore different configuration spaces. In order to apply the method described above for the computation of $\Delta F(\beta, L, A)$ one identifies the initial Hamiltonian $H_{1}$ and the configuration space $\mathcal{C}$ with the corresponding ones of the model on the lattice 

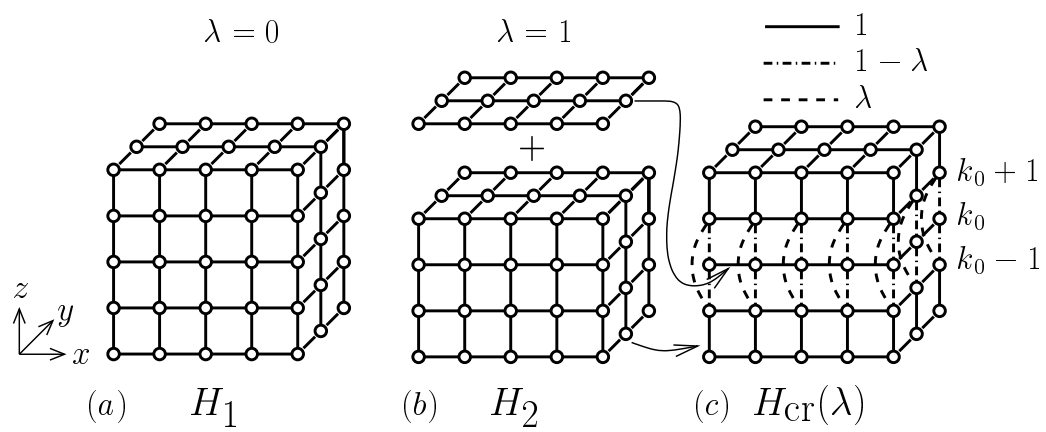

FIG. 1: Bond arrangement for the computation of the free energy difference in eq. (5) (see main text).

$A \times L$, as depicted in fig. 1(a). Accordingly, $F_{1}(\beta, L, A)=F(\beta, L, A)$. The configuration space of the final system can be arranged to be equal to $\mathcal{C}$ by adding to the model on the lattice $A \times(L-1)$ we are actually interested in a two-dimensional lattice of size $A$ with suitable degrees of freedom and lateral PBC (see fig. $1(\mathrm{~b})$ ). The final Hamiltonian $\mathrm{H}_{2}$ is then constructed such that the added layer does not interact with the remaining part of the system and therefore $F_{2}(\beta, L, A)=F(\beta, L-1, A)+F_{2 D}(\beta, A)$, where $F_{2 D}(\beta, A)$ is the free energy of the isolated two-dimensional layer. Although the argument is quite general, we focus now on the case of interest in which the lattice degrees of freedom (e.g., spins of the Ising model) interact only with their nearest neighbours on the same lattice, with a coupling strength $J=1$ (indicated by solid bonds in figs. 1 (a) and (b)). The crossover Hamiltonian $H_{\text {cr }}(\lambda)$ (see eq. (3)) additionally depends on the position $k_{0} \in\{1,2, \ldots, L\}$ (along the $z$ direction) of the two-dimensional layer which decouples from the rest of the system upon passing from $\lambda=0$ to $\lambda=1$, i.e., from fig. (1)(a) to (b). The resulting $H_{\text {cr }}(\lambda)$ is characterized by the coupling constants depicted in fig. 团(c) whereas $\Delta H$ (see eq. (5)) can be determined as $\Delta H=H_{\mathrm{cr}}(\lambda=1)-H_{\mathrm{cr}}(\lambda=0) . \Delta F$ (see eqs. (2) and (5)) can be finally expressed as $\Delta F(\beta, L, A)=-I(\beta, L, A)+F_{2 D}(\beta, A)$ from which one has still to subtract $f^{\text {bulk }}(\beta)$ in order to determine the Casimir force in a slab of thickness $L-1 / 2$ (see eq. (2) ). However, it is numerically more convenient to avoid the computation of $f^{\text {bulk }}(\beta)$ by considering, instead, the difference between the Casimir forces in slabs of thicknesses $L_{1}$ and $L_{2}>L_{1}$ :

$$
\begin{aligned}
\Delta \hat{f}_{\mathrm{C}}\left(\beta, L_{1}, L_{2}, A\right) & \equiv \hat{f}_{\mathrm{C}}\left(\beta, L_{1}-\frac{1}{2}, A\right)-\hat{f}_{\mathrm{C}}\left(\beta, L_{2}-\frac{1}{2}, A\right) \\
& =\beta A^{-1}\left[I\left(\beta, L_{1}, A\right)-I\left(\beta, L_{2}, A\right)\right],
\end{aligned}
$$

in which the contributions of both $f^{\text {bulk }}(\beta)$ and $F_{2 D}(\beta, A)$ actually cancel. Below we de- 
scribe the method used to determine $\vartheta$ in eq. (1) on the basis of the numerical data for $\Delta \hat{f}_{\mathrm{C}}\left(\beta, L_{1}, L_{2}, A\right)$. In passing, we note that although $H_{\mathrm{cr}}(\lambda)$ (see fig. 1 (c)), $\Delta H$, and therefore $\langle\Delta H\rangle_{H_{\mathrm{cr}}(\lambda)}$ depend on the choice of $k_{0}, \int_{0}^{1} \mathrm{~d} \lambda\langle\Delta H\rangle_{H_{\mathrm{cr}}(\lambda)}$ is actually independent of it, as long as the boundary conditions are not affected by the extraction of the $k_{0}$-th layer. In particular, imposing the $\mathrm{BC}$ at the boundary layers in the $z$-direction, this requires $k_{0} \neq 1, L$ for fixed and open BC, whereas for PBC there is no restriction and indeed translational invariance implies that $\langle\Delta H\rangle_{H_{\mathrm{cr}}(\lambda)}$ is actually independent of $k_{0}$. In our simulations we have taken $k_{0}=L / 2$.

Within the MC simulations we compute the ensemble averages $\langle\Delta H\rangle_{H_{\mathrm{cr}}(\lambda)}$ for different values of $\beta$, lattice sizes, and $\lambda$. Then, via numerical integration (Simpsons method with 20 points) we calculate the integral $I(\beta, L, A)$ in eq. (5) and thus $\Delta \hat{f}_{\mathrm{C}}\left(\beta, L_{1}, L_{2}, A\right)$ (see eq. (6) ) with $\left(L_{1}, L_{2}\right)=(L, 2 L)$ and $L=13,16,20$ for the Ising model, whereas $L=10,15,20$ for the XY model. For a given pair of thicknesses $\left(L_{1}, L_{2}\right)$, fixed $A$ and $\mathrm{BC}$, the scaling function $\vartheta$ of the Casimir force can be extracted from the temperature dependence of $\Delta \hat{f}_{\mathrm{C}}$ by using the fact that, for large $L_{1,2}$ and $A, \hat{f}_{\mathrm{C}}$ in eq. (6) scales according to eq. (1). In particular, it is useful to focus on the quantity

$$
g\left(y ; L_{1}, L_{2}, A\right) \equiv\left(L_{1}-1 / 2\right)^{d} \Delta \hat{f}_{\mathrm{C}}\left(\beta\left(y ; L_{1}\right), L_{1}, L_{2}, A\right),
$$

as a function of $y$, where $\beta\left(y ; L_{1}\right) \equiv \beta_{c} /\left[1+y\left(L_{1}-1 / 2\right)^{-1 / \nu}\right]$ and $d=3 ; g$ is expected to scale as (see eq. (1))

$$
g\left(y ; L_{1}, L_{2}, A\right)=\hat{\theta}(y)-\alpha^{-d} \hat{\theta}\left(\alpha^{1 / \nu} y\right),
$$

where $\alpha=\left(L_{2}-1 / 2\right) /\left(L_{1}-1 / 2\right)$ is the width ratio and $\hat{\theta}(y)$ is the lattice estimate of $\theta(y) \equiv \vartheta\left(y /\left(\xi_{0}^{+}\right)^{1 / \nu}\right)$. For given $g$ eq. (8) can be solved for $\hat{\theta}(y)$ via an iterative method. In the first step one takes $\hat{\theta}_{0}(y) \equiv g\left(y ; L_{1}, L_{2}, A\right)$ as a first approximation of the actual $\hat{\theta}$. In turn, this approximant can be improved by taking into account that eq. (8) yields $\hat{\theta}(y)=\hat{\theta}_{0}(y)+\alpha^{-d} \hat{\theta}\left(\alpha^{1 / \nu} y\right) \simeq \hat{\theta}_{0}(y)+\alpha^{-d} \hat{\theta}_{0}\left(\alpha^{1 / \nu} y\right)$, so that a better approximant $\hat{\theta}_{1}(y)$ is provided by $\hat{\theta}_{1}(y)=\hat{\theta}_{0}(y)+\alpha^{-d} \hat{\theta}_{0}\left(\alpha^{1 / \nu} y\right)$ where the value of $\hat{\vartheta}_{0}$ at the point $\alpha^{1 / \nu} y$ is obtained by cubic spline interpolation of the available data. In the expression for $\hat{\theta}_{1}$ one can replace $\hat{\theta}_{0}$ by using eq. (8), yielding $\hat{\theta}_{1}(y)=\hat{\theta}(y)-\alpha^{-2 d} \hat{\theta}\left(\alpha^{2 / \nu} y\right)$, which, in turn, can be solved as already done before for eq. (8) by introducing $\hat{\theta}_{2}(y)=\hat{\theta}_{1}(y)+\alpha^{-2 d} \hat{\theta}_{1}\left(\alpha^{2 / \nu} y\right)=$ $\hat{\theta}(y)-\alpha^{-4 d} \hat{\theta}\left(\alpha^{4 / \nu} y\right)$, and so on. This iterative procedure yields a sequence of approximants $\hat{\theta}_{k \geq 1}(y)=\hat{\theta}_{k-1}(y)+\alpha^{-2^{k-1} d} \hat{\theta}_{k-1}\left(\alpha^{2^{k-1} / \nu} y\right)$, which converges very rapidly because the correc- 
tion to the $k$-th approximant is of the order of $\alpha^{-2^{k-1} d}$, i.e., exponentially small in $2^{k}$. Already for $k=5$ one has $\alpha^{-2^{k-1} d} \simeq 3.5 \times 10^{-15}$ in $3 \mathrm{D}$ with $\alpha \simeq 2([24])$. Accordingly, we approximate $\hat{\theta}(y) \equiv \hat{\theta}_{k \rightarrow \infty}(y)$ by $\hat{\theta}_{5}(y)$. The result for the universal scaling function $\hat{\vartheta}(x) \equiv \hat{\theta}\left(x\left(\xi_{0}^{+}\right)^{1 / \nu}\right)$ of $\hat{f}_{\mathrm{C}}$, as obtained from a specific pair of lattices with thicknesses $(L, 2 L)$, should be independent of the actual value of $L$, at least for large $L$. However, for the thicknesses we used in our MC simulations, corrections to the leading scaling behaviour are actually relevant [16] and affect both the scaling variable

$$
x \equiv \tau\left(L / \xi_{0}^{+}\right)^{1 / \nu}\left(1+g_{\omega} L^{-\omega}\right)
$$

and the scaling function $\hat{\vartheta}(x)$ which additionally depends on $L^{-\omega^{\prime}}: \hat{f}_{\mathrm{C}}(\beta, L, A)=$ $L^{-d} \hat{\vartheta}\left(x, L^{-\omega^{\prime}}\right) \simeq L^{-d} \vartheta(x)\left[1+L^{-\omega^{\prime}} \phi(x)+\ldots\right]$ for large $L$. In eq. (9), $\omega$ is the leading correction-to-scaling exponent $\omega \simeq 0.84(4)$ and 0.79 [17] for the Ising and XY UC, respectively, in $3 \mathrm{D} . \omega^{\prime}$ controls the leading corrections to scaling of the estimator $\hat{f}_{\mathrm{C}}$ and its value, typically equal to $\omega$, can be increased by using suitably improved Hamiltonians and observables [17] so that these corrections are reduced. However, we point out that in the present case surface operators [6] might even yield $\omega^{\prime}<\omega$. For small lattice sizes, next-toleading corrections to scaling (e.g., $\sim L^{-1}$ ) might also be of relevance, resulting in effective $L$-dependent exponents. A detailed analysis of all these corrections and the determination of $\phi(x)$ and $\omega^{\prime}$ is beyond the scope of the present paper and requires the study of much larger lattices. As a phenomenological ansatz for the effective corrections we take $\omega^{\prime} \simeq \omega \simeq 1$ and [16]

$$
\hat{f}_{\mathrm{C}}(\beta, L, A)=L^{-d}\left(1+g_{1} L^{-1}\right)^{-1} \vartheta(x)
$$

which, for suitable choices of $g_{1}$ and $g_{\omega}$, yields a good data collapse of the curves corresponding to different sizes. We point out that an equally satisfactory data collapse can be obtained - within the range of the scaling variable $x$ explored here - by assuming a different functional form for the corrections. The resulting estimate of $\vartheta(x)$ is slightly affected by this choice and only larger scale simulations can provide an estimate of $\vartheta(x)$ which is unbiased in this respect. In addition to these corrections to scaling, the simulation data depend on the aspect ratio $\rho \equiv L / \sqrt{A}$. Whereas in the case of the XY model this dependence is quite pronounced [16], for the Ising model with $(++)$ and $(+-)$ BC and for $x \geq-6, \Delta \hat{f}_{\mathrm{C}}(\beta, L, 2 L, A)$ exhibits only a very weak dependence on $\rho$ already for $\rho \leq 1 / 6$, as we have tested by considering lattices with $1 / \rho=6,10,14$. The results we present here for 
the Ising model refer to lattices with fixed $\rho=1 / 6$. For the XY model we have accounted for corrections due to $\rho \neq 0$ in accordance with ref. [16] by considering multiplicative corrections $1+r_{1} \rho^{2}$ and $\left(1+r_{2} \rho^{2}\right)^{-1}$ to $x$ and $\hat{f}_{\mathrm{C}}$, respectively, which allow the extrapolation of the data on lattices with $1 / \rho=4,6,8$ to $\rho \rightarrow 0$.

The computation of the canonical average $\langle\Delta H\rangle_{H_{\mathrm{cr}}(\lambda)}$ has been carried out via an hybrid MC method in which a suitable mixture of Wolff and Metropolis algorithms is used [21]. In particular, for the Ising model each hybrid MC step consists of one flip of a Wolff cluster according to the Wolff algorithm, typically followed by $9 A$ attempts to flip a spin $s_{x, y, z}$ with $z \in\left\{k_{0}-1, k_{0}, k_{0}+1\right\}$, which are accepted according to the Metropolis rate [21]. An analogous method, with a suitable implementation of Metropolis and Wolff algorithms has been used for the XY model [19]. We tested our MC program successfully by comparing $g(y, 5,10,9)$ with corresponding transfer-matrix data.

\section{3D XY MODEL}

We have determined the scaling function of the Casimir force in the 3D XY model with free $\mathrm{BC}$, which is of relevance for the ${ }^{4} \mathrm{He}$ experiment mentioned in the introduction. The resulting scaling function is plotted in fig. 2, In order to achieve scaling we have accounted for corrections to scaling according to eq. (10) with $g_{1}=6.4(2), g_{\omega}=2.1(2)$ and for the corrections due to $\rho \neq 0$ in accordance with ref. [16] with $r_{1}=2.3(2)$ and $r_{2}=1.1(1)$ (see ref. [16] for details). The scaling function in fig. 2] is compatible, within the errorbars, with the one determined in ref. [16], providing an independent test both of the results presented there and of our method to compute it. In the approach used in ref. [16], $\hat{f}_{\mathrm{C}}$ is computed via the internal energy density $u$ and an integration over the temperature, whereas here this is carried out via the free energy density $f$ and an integration over the coupling $\lambda$. In ref. [16] one takes advantage of the possibly available numerical knowledge of the bulk energy density $u^{\text {bulk }}$ of the model of interest whereas here the analogous information on $f^{\text {bulk }}$ is not required for the determination of $\hat{\vartheta}$, making our approach applicable also

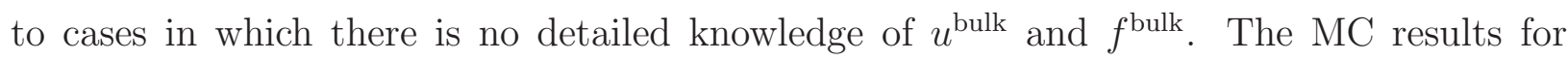
$\vartheta(x)$ in fig. 2 compare also very well with the experimental data in ref. [13] (we have used the experimental value $\xi_{0}^{+(\exp )}=1.432 \AA$ for the normalization of $x$ ). In particular with $x_{\min }=-5.29(7)$ and $\vartheta_{\min } \equiv \vartheta\left(x_{\min }\right)=-1.41(2)$, it captures properly the corresponding 


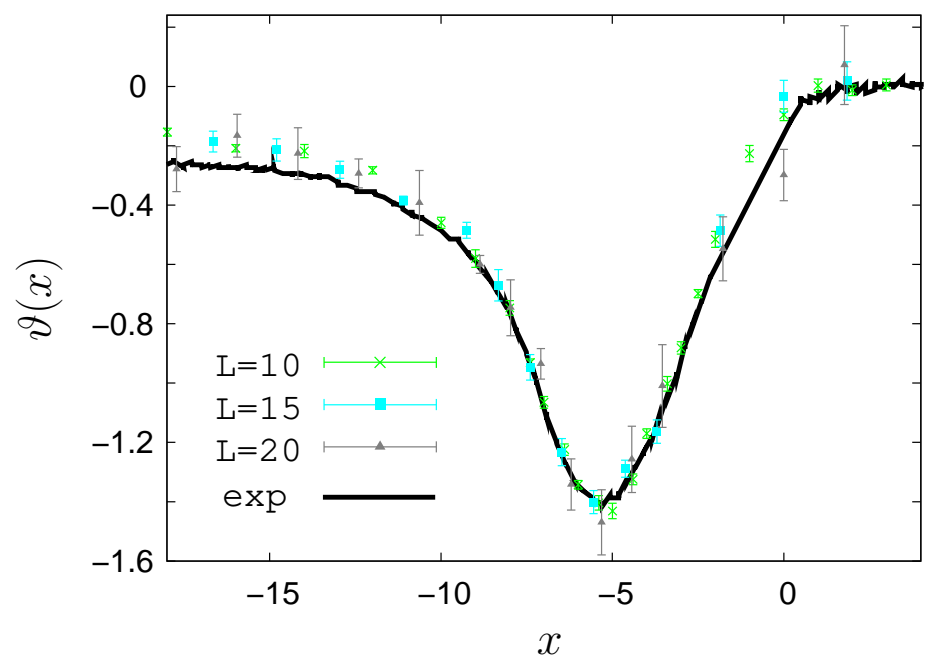

FIG. 2: Scaling function $\vartheta$ of the Casimir force for the 3D XY bulk UC and so-called ordinary surface UC corresponding to free boundary conditions. Our MC data compare very well with the corresponding experimental data from ref. [13] (solid line).

experimental values $x_{\min }^{(\exp )}=-5.7(5)$ and $\vartheta_{\min }^{(\exp )}=-1.30(3)$ for the pronounced minimum. In comparing $\vartheta_{\min }$ with $\vartheta_{\min }^{(\exp )}$ one has to take into account that, as pointed out above, the numerical determination of $\vartheta$ is actually influenced by the choice of the ansatz for the corrections to scaling. Indeed, replacing the multiplicative correction $\left(1+g_{1} L^{-1}\right)^{-1}$ in eq. (10) with one of the form $\left(1-\tilde{g}_{1} L^{-1}\right)$ (which is equivalent to the previous one for large $L)$, the resulting scaling function displays a good data collapse for $\tilde{g}_{1}=3.1(1)$. It has the same shape as the one in fig. 2 but its overall amplitude is reduced by a factor $R \simeq 0.89$. With this caveat, our estimates for $x_{\min }$ and $\vartheta_{\min }$ are compatible also with those of ref. [16] $(-5.3(1)$ and $-1.35(3)$, respectively).

\section{3D ISING MODEL}

For this bulk UC we have determined the scaling functions $\vartheta$ for three different BC: $(+-)$, $(++)$ (pairs of lattices with sizes $(L, 2 L)$ and $L=13,16,20)$ and $\operatorname{PBC}(L=10,16,20)$. The results are reported in figs. 3, 4 and 5, respectively. Each data point has been averaged over at least $10^{5}$ hybrid MC steps. The scaling function in fig. 3 has been obtained accounting for the corrections according to eq. (10) with $g_{1}=14.8(2)$ and $g_{\omega}=2.9(2)$. The resulting data collapse is very good and only at very low temperatures corrections to scaling are stronger 


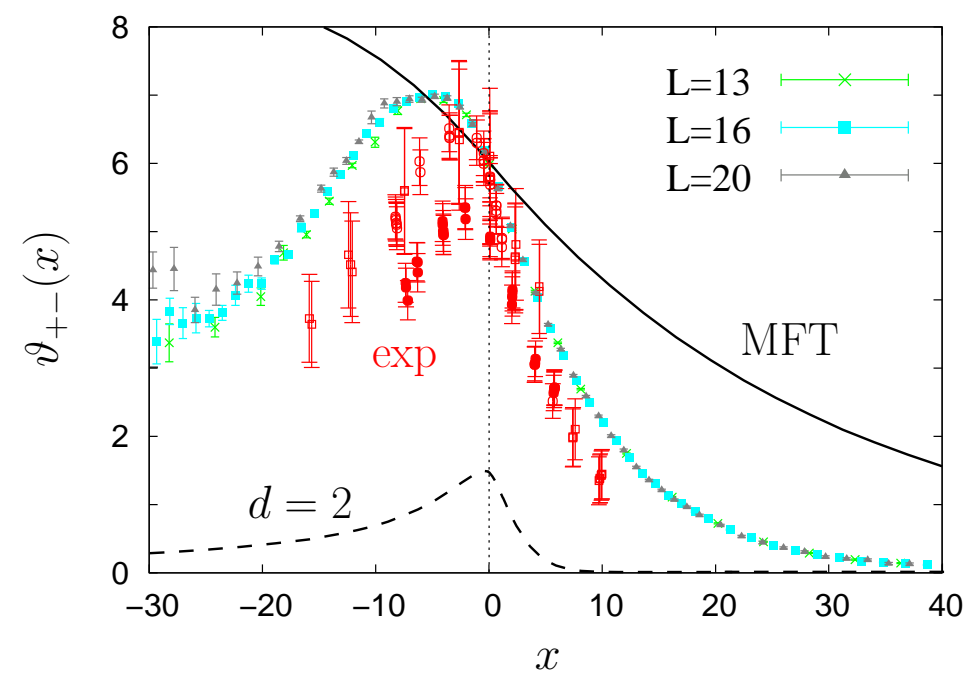

FIG. 3: Scaling function $\vartheta_{+-}$of the Casimir force in the 3D Ising model with $(+-)$boundary conditions, compared with the mean-field prediction (solid line), the experimental data of ref. [9] and the exact result for the two-dimensional Ising model (dashed line).

and not fully accounted for by the ansatz in eq. (10). Such stronger corrections might be related to the presence of an interface in the system. $\vartheta_{+-}$is compared with the experimental data in ref. [9], the prediction of mean-field theory [10] (solid line, normalized such that $\left.\vartheta_{+-}^{(\mathrm{MFT})}(0)=\vartheta_{+-}^{(\mathrm{MC})}(0)\right)$ and the corresponding result for the two-dimensional Ising model [12] (dashed line). From the data set with $L=13$ we estimate $\vartheta_{+-}(0)=5.97(2)$, in agreement with the experimental value $\vartheta_{+-}^{(\exp )}(0)=6(2)$ [9] but which is larger compared to the previous MC estimate $\vartheta_{+-}(0)=4.900(64)[10]$ and the analytical estimates $\vartheta_{+-}^{(\mathrm{FT})}(0)=3.16,4.78$. The latter depend on the approximant used to resum the field-theoretical $\epsilon=4-d$-expansion up to $O(\epsilon)$ (see ref. [10] for details). A good data collapse is obtained also for $\tilde{g}_{1}=5.0(1)$ which, compared to fig. 3, yields an overall reduction of the amplitude of $\vartheta_{+-}$by a factor $R \simeq 0.76$. In addition, we expect the experimental data in ref. [9] to be affected by corrections to scaling already for $x \gtrsim 2$, due to the relatively small corresponding value of $\xi / \ell \lesssim 30$, where $\ell \simeq 3 \AA$ is the molecular scale in the experiment. In view of these difficulties, the agreement between the $\mathrm{MC}$ and the experimental data in fig. 3 is encouraging. In fig. 4 the scaling function $\vartheta_{++}$for $(++)$BC has been obtained accounting for the corrections in eq. (10) with $g_{1}=14.2(7)$ and $g_{\omega}=2.3(2)$. Using, instead, $\tilde{g}_{1}=4.9(2)$ yields $R \simeq 0.77$. Currently, for this $\mathrm{BC}$ and in film geometry no experimental data are available for comparison, but $\vartheta_{++}$can be compared with the prediction of mean-field theory [10] (solid line, normalized as before) 


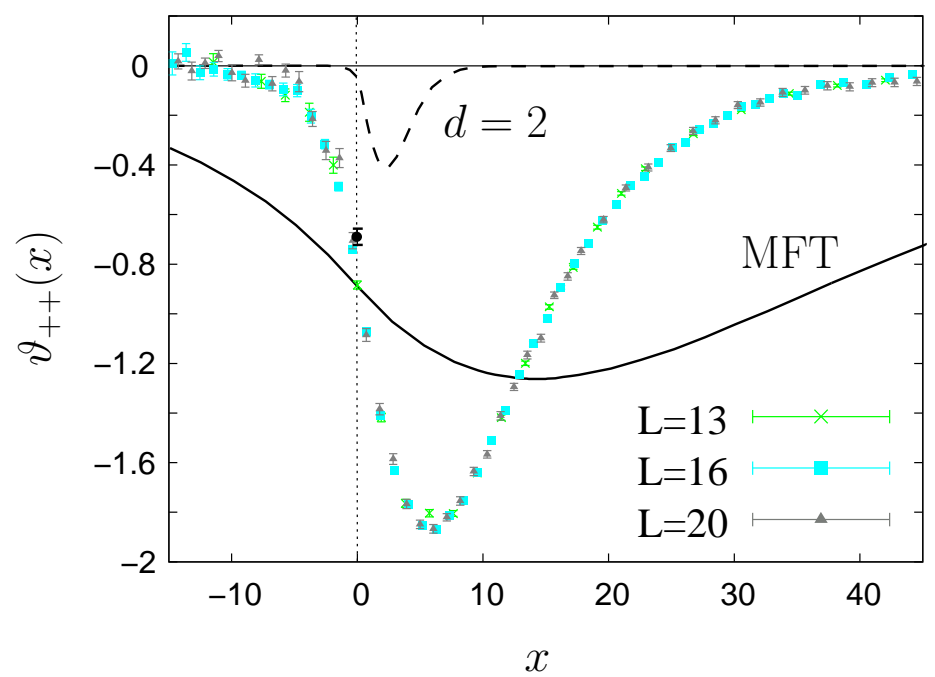

FIG. 4: Scaling function $\vartheta_{++}$of the Casimir force in the 3D Ising model with $(++)$boundary conditions, compared with the mean-field prediction (solid line) and the exact result for the twodimensional Ising model (dashed line); • MC [10].

and of the two-dimensional Ising model [12] (dashed line). From the data with $L=13$ we estimate $\vartheta_{++}(0)=-0.884(16)$ which is slightly larger than the previous MC estimate $\vartheta_{++}(0)=-0.690(32)$ [10] (indicated as a black dot in fig. 4, still affected by finite-size corrections) and the corresponding field-theoretical predictions $\vartheta_{++}^{(\mathrm{FT})}(0)=-0.652,-0.346$ depending on the approximant used to resum the $O(\epsilon)$ series (see ref. [10] for details).

For PBC and $L \geq 10$ corrections to scaling turn out to be negligible and the resulting scaling function $\vartheta_{\mathrm{P}}$ in fig. 5 is in very good agreement with its previous determination in ref. [8] based on the computation of the lattice stress tensor. The slight discrepancies might be due to the uncertainty in the normalization factor which had to be used in ref. [8]. This agreement provides additional support concerning the reliability of our approach. Figure 5 shows also the comparison with the available field-theoretical predictions [7] (solid and dashed lines) for $x \geq 0$ up to $O(\epsilon)$. The discrepancies can be traced back to higher-order nonanalytic contributions $\sim \epsilon^{3 / 2}[22]$.

\section{CONCLUSIONS}

We have presented a novel general approach to determine the universal scaling functions $\vartheta$ of Casimir forces via Monte Carlo simulations. The corresponding results for the three- 


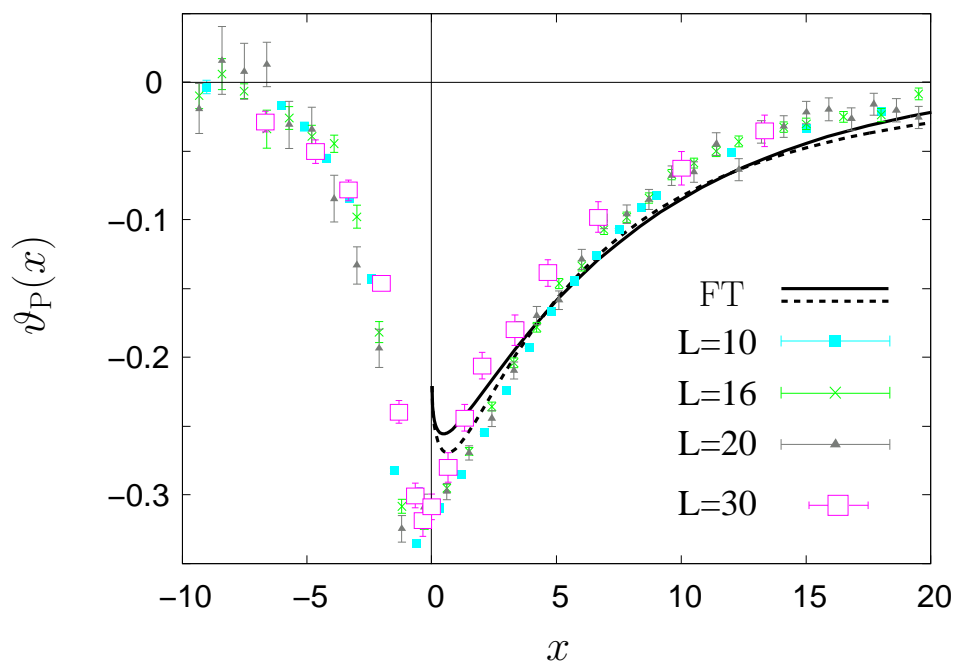

FIG. 5: Scaling function $\vartheta_{\mathrm{P}}$ of the Casimir force in the 3D Ising model with PBC. For comparison we report the normalized data set with $L=30$ from ref. [8] which corresponds to the largest lattice size investigated therein. The solid (dashed) curve corresponds to the $[1,0]([0,1])$ Padé approximant of the analytical prediction in refs. [3, 7].

dimensional Ising and XY UCs compare favourably with previous experimental, numerical and analytical results. Our predictions for $\vartheta_{++}$and $\vartheta_{+-}$in a film geometry might also contribute to the understanding of Casimir forces acting on colloidal particles.

\section{Acknowledgments}

The authors acknowledge the important contribution of M. De Prato to the early stages of this work. They are grateful to A. Hucht, M. Krech and E. Vicari for useful discussions and to M. Fukuto and R. Garcia for providing the experimental data.

[1] Casimir H. B., Proc. K. Ned. Akad. Wet. 51 (1948) 793.

[2] Kardar M. and Golestanian M., Rev. Mod. Phys. 71 (1999) 1233; Ball P., Nature 447 (2007) 772 .

[3] Krech M., Casimir Effect in Critical Systems (World Scientific, Singapore, 1994); J. Phys.: Condens. Matter 11 (1999) R391. 
[4] Hanke A., Schlesener F., Eisenriegler E. and Dietrich S., Phys. Rev. Lett. 81 (1998) 1885; Schlesener F., Hanke A. and Dietrich S., J. Stat. Phys. 110 (2003) 981.

[5] Fisher M. E. and de Gennes P. G., C. R. Acad. Sci. Paris Ser. B 287 (1978) 207.

[6] Diehl H. W., Phase Transitions and Critical Phenomena, edited by Domb C. and Lebowitz J. L., Vol. 10 (Academic, London, 1986) p. 76.

[7] Krech M. and Dietrich S., Phys. Rev. A 46 (1992) 1886; 46 (1992) 1922.

[8] Dantchev D. and Krech M., Phys. Rev. E 69 (2004) 046119.

[9] Fukuto M., Yano Y. F. and Pershan P. S., Phys. Rev. Lett. 94 (2005) 135702.

[10] Krech M., Phys. Rev. E 56 (1997) 1642.

[11] Borjan Z. and Upton P. J., Phys. Rev. Lett. 81 (1998) 4911.

[12] Evans R. and Stecki J., Phys. Rev. B 49 (1994) 8842.

[13] Garcia R. and Chan M. H. W., Phys. Rev. Lett. 83 (1999) 1187; Ganshin A., Scheidemantel S., Garcia R. and Chan M. H. W., Phys. Rev. Lett. 97 (2006) 075301.

[14] Zandi R., Rudnick J. and Kardar M., Phys. Rev. Lett. 93 (2004) 155302.

[15] Maciołek A., Gambassi A. and Dietrich S., Phys. Rev. E in print, arXiv:0705.1064; Zandi R., Shackell A., Rudnick J., Kardar M. and Chayes L. P., preprint arXiv:cond-mat/0703262.

[16] Hucht A., preprint arXiv:0706.3458.

[17] Pelissetto A. and Vicari E., Phys. Rep. 368 (2002) 549.

[18] Ruge C., Zhu P. and Wagner F., Physica A 209 (1994) 431.

[19] Gottlob A. P. and Hasenbusch M., Physica A 201 (1993) 593.

[20] Mon K. K., Phys. Rev. B 39 (1989) 467; Mon K. K. and Binder K., Phys. Rev. B 42 (1990) 675 .

[21] Landau D. P. and Binder K., A Guide to Monte Carlo Simulations in Statistical Physics (Cambridge University Press, London, 2005) p. 155.

[22] Diehl H. W., Grüneberg D. and Shpot M. A., Europhys. Lett. 75 (2006) 241.

[23] Note that although the values of $\xi_{0}^{+}$quoted here refer to the second moment correlation length $\xi_{2^{\text {nd }}}, \xi / \xi_{2^{\text {nd }}} \simeq 1$ for $\beta<\beta_{c}$ for both the Ising and the XY model [17, 19].

[24] Note that a smaller value of $\alpha$ reduces the sizes of the lattices required for the computation of $\hat{f}_{\mathrm{C}}$. On the other hand, such a choice decreases the accuracy of the iterative procedure to determine $\hat{\theta}$. 